\title{
Pairing matrix elements and pairing gaps with bare, effective, and induced interactions
}

\author{
F. Barranco, ${ }^{1}$ P. F. Bortignon, ${ }^{2,3}$ R. A. Broglia, ${ }^{2,3,4}$ G. Colò,${ }^{2,3}$ P. Schuck,${ }^{5}$ E. Vigezzi, ${ }^{3}$ and X. Viñas ${ }^{6}$ \\ ${ }^{1}$ Departamento de Fisica Aplicada III, Escuela Superior de Ingenieros, Universidad de Sevilla, Camino de los Descubrimientos s/n, \\ E-41092 Sevilla, Spain \\ ${ }^{2}$ Dipartimento di Fisica, Università degli Studi, via Celoria 16, I-20133 Milano, Italy \\ ${ }^{3}$ INFN Sezione di Milano, via Celoria 16, I-20133 Milano, Italy \\ ${ }^{4}$ The Niels Bohr Institute, University of Copenhagen, Blegdamsvej 17, DK-20100 Copenhagen Ø, Denmark \\ ${ }^{5}$ Institut de Physique Nucléaire, 15 rue Georges Clémenceau, F-91406 Orsay Cedex, France \\ ${ }^{6}$ Departament d' Estructura i Constituents de la Matèria, Facultat de Fìsica, Universitat de Barcelona, Diagonal 647, \\ E-08028 Barcelona, Spain
}

(Received 24 February 2005; published 21 November 2005)

\begin{abstract}
The dependence on the single-particle states of the pairing matrix elements of the Gogny force and of the

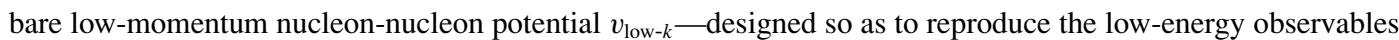
avoiding the use of a repulsive core-is studied for a typical finite, superfluid nucleus $\left({ }^{120} \mathrm{Sn}\right)$. It is found that the matrix elements of $v_{\text {low- }}$ follow closely those of $v_{\text {Gogny }}$ on a wide range of energy values around the Fermi energy $e_{F}$, those associated with $v_{\text {low- } k}$ being less attractive. This result explains the fact that around $e_{F}$ the pairing gap $\Delta_{\text {Gogny }}$ associated with the Gogny interaction (and with a density of single-particle levels corresponding to an effective $k$ mass $m_{k} \approx 0.7 \mathrm{~m}$ ) is a factor of about 2 larger than $\Delta_{\text {low- } k}$, being in agreement with $\Delta_{\exp }=$ 1.4 MeV. The exchange of low-lying collective surface vibrations among pairs of nucleons moving in time-reversal states gives rise to an induced pairing interaction $v_{\text {ind }}$ peaked at $e_{F}$. The interaction $\left(v_{\text {low }-k}+v_{\text {ind }}\right) Z_{\omega}$ arising from the renormalization of the bare nucleon-nucleon potential and of the single-particle motion $(\omega$-mass and quasiparticle strength $Z_{\omega}$ ) associated with the particle-vibration coupling mechanism, leads to a value of the pairing gap at the Fermi energy $\Delta_{\text {ren }}$ that accounts for the experimental value. An important question that remains to be studied quantitatively is to what extent $\Delta_{\text {Gogny }}$, which depends on average parameters, and $\Delta_{\text {ren }}$, which explicitly depends on the parameters describing the (low-energy) nuclear structure, display or not a similar isotopic dependence and whether this dependence is borne out by the data.
\end{abstract}

DOI: 10.1103/PhysRevC.72.054314

PACS number(s): 21.30.Fe, 21.60.-n, 27.60.+j

\section{INTRODUCTION}

An economic description of pairing correlations in finite nuclei is provided by Hartree-Fock-Bogoliubov (HFB) theory $[1,2]$, making use of phenomenological interactions such as the finite-range Gogny force [3] or density-dependent zero-range forces combined with appropriate renormalization procedures or energy cutoffs (e.g., Refs. [4-6]). Such a description leads to values of the pairing gap that are in overall agreement with the experimental findings. Note that in these calculations, the density of levels at the Fermi energy $\rho\left(e_{F}\right)$ is controlled by the so-called $k$-mass [i.e., $\rho\left(e_{F}\right) \sim m_{k}$ ], which, as a rule, is smaller than the bare nucleon mass (e.g., $m_{k} \approx 0.7 m$ in the case of the Gogny force).

Conversely, a number of studies have shown that the superfluid properties of nuclear systems, ranging from infinite nuclear and neutron matter to finite atomic nuclei, are strongly influenced by polarization phenomena [7-9]. In these calculations one starts from a bare nucleon-nucleon potential (Argonne, Bonn, Paris, etc.) adding afterwards the renormalization processes. Recently, the pairing gap, the quasiparticle spectrum and the collective modes of ${ }^{120} \mathrm{Sn}$ have been calculated solving the Dyson-Gor'kov equation [8], in a single-particle space characterized by $m_{k}=0.7 \mathrm{~m}$, allowing the nucleons to interact in the ${ }^{1} S_{0}$ channel through a $v_{14}$ Argonne $N N$ potential taking into account the variety of renormalization processes (self-energy, fragmentation, induced interaction, and vertex corrections) arising from the coupling of the particles with surface vibrations. While the bare $N N$ interaction accounts for about half of the pairing gap, overall agreement with the experimental findings is achieved by including medium polarization effects.

In the present article we want to shed light into the physics of these results, by studying the magnitude of the different pairing $\left(J^{\pi}=0^{+}\right)$matrix elements as well as their dependence on the energy of the single-particle states lying in the vicinity of the Fermi energy in Sec. II and by discussing the calculation of the pairing gaps associated with these matrix elements in Sec. III.

\section{MATRIX ELEMENTS}

The matrix element of the induced interaction can be written as follows [9]:

$\left\langle v^{\prime} \bar{v}^{\prime}\left|v_{\text {ind }}\right| v, \bar{v}\right\rangle=2 \sum_{L M n} \frac{\left\langle v\left|f_{L n} Y_{L M}\right| v^{\prime}\right\rangle\left\langle\bar{v}\left|f_{L n} Y_{L M}^{*}\right| \bar{v}^{\prime}\right\rangle}{E_{0}-\left|e_{\nu}-e_{F}\right|-\left|e_{v^{\prime}}-e_{F}\right|-\hbar \omega_{L n}}$,

where $L, M$, and $n$ denote the quantum numbers of the exchanged collective vibrations, $f_{L n}(r)=\beta_{L n} R_{0}(d U / d r)$ is the associated radial form factor $\left(\beta_{L n}\right.$ being the deformation parameter associated with the $n$th mode of multipolarity $L, R_{0}$ 

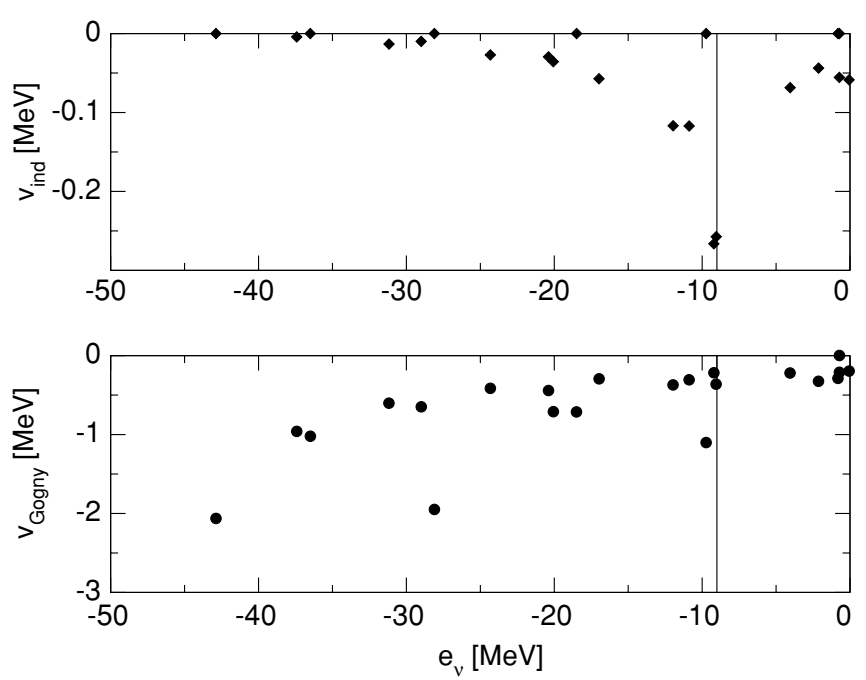

FIG. 1. The nucleus ${ }^{120} \mathrm{Sn}$. Diagonal pairing matrix elements of the induced interaction (upper panel, solid diamonds) and of the Gogny force (lower panel, solid circles), displayed as a function of the single-particle energy, $e_{v}$, of the state $v$ calculated using the bare nucleon mass and the single-particle wave functions of a WoodsSaxon potential with standard parameters (depth $V_{0}=-49 \mathrm{MeV}$, diffusivity $a=0.65 \mathrm{fm}$, and radius $R_{0}=6.16 \mathrm{fm}$ ), including the spin-orbit term, parametrized according to Ref. [21]. Also shown by means of vertical lines is the position of the Fermi energy, $e_{F}=$ $-9.1 \mathrm{MeV}$. Note the different scale in the two figures.

the ground-state radius, and $U$ the average potential), $\hbar \omega_{L n}$ is the energy of the $n$th vibrational mode, and $e_{v}$ and $e_{F}$ are the single-particle and Fermi energies. $E_{0}$ is the pairing correlation energy per Cooper pair, which is of the order of $-\Delta$. In practice we have used $E_{0}=-2 \mathrm{MeV}$. The prefactor 2 in Eq. (1) comes from the two possible time orderings associated with the one-phonon exchange (cf. Fig. 10(f)).

In the upper panel of Fig. 1 we show the value of the diagonal matrix elements $\left\langle\nu \bar{\nu}\left|v_{\text {ind }}\right| \nu \bar{v}\right\rangle$ of the induced interaction for the nucleus ${ }^{120} \mathrm{Sn}$ as a function of the single-particle energy. Also shown in the lower panel of Fig. 1 are the diagonal matrix elements of the Gogny interaction. Here, and in the rest of the article, we employ the D1 parametrization. The single-particle levels needed to obtain these matrix elements have been calculated making use of a standard Woods-Saxon potential, whose parameters are provided in the caption of Fig. 1. In most of our calculations we use this potential and an associated effective mass $m_{k}$ equal to the bare mass. In the last section we use instead an effective mass $m_{k}=0.7 \mathrm{~m}$, which simulates the typical outcome of a Hartree-Fock calculation (with, e.g., the Gogny interaction). In those cases, the Woods-Saxon parameters are changed in such a way that the Fermi energy remains close to the experimental value $\left(V_{0}^{\prime} \approx\left(m / m_{k}\right) V_{0}\right.$, see, e.g., Fig. 8). Note that $m_{k}$ is, as a rule, a smooth function of $r$ [10]. The parameters $\beta_{L n}$ and $\hbar \omega_{L n}$ have been determined by diagonalizing a separable multipole-multipole interaction in the quasiparticle-random phase approximation (QRPA), adjusting the coupling constants to reproduce the energies and transition probabilities of the lowest lying states of each spin.
The matrix elements of $v_{\text {ind }}$, which display an average value equal to $-0.15 \mathrm{MeV}$, are peaked at the Fermi energy, within an energy range of essentially $5 \mathrm{MeV}$ around $e_{F}$. This behavior reflects the fact that $\left\langle v^{\prime} \bar{v}^{\prime}\left|v_{\text {ind }}\right| \nu, \bar{v}\right\rangle$ is controlled, through the energy denominator appearing in Eq. (1), by the energy of the low-lying collective vibrations $\left[\hbar \omega_{L_{(n)}}<5 \mathrm{MeV}\right]$. Outside this energy range, the values of $\left\langle v^{\prime} \bar{v}^{\prime}\left|v_{\text {ind }}\right| v, \bar{v}\right\rangle$ become very small, less than $50 \mathrm{keV}$ in absolute value. The matrix elements of $v_{\text {Gogny }}$ decrease on average smoothly in magnitude all the way from the deepest levels up to the continuum threshold, displaying a value of about $-0.3 \mathrm{MeV}$ at $e_{F}$.

The induced interaction matrix elements depend on specific properties of finite nuclei, namely the single-particle quantum numbers and energies, and the energy, the zero-point amplitude, and the transition density of the vibrational states. To study the global features of the bare, of the induced, and of the Gogny interactions as well as to provide a more transparent form for their matrix elements, we also present, in the rest of this section, the matrix elements obtained using the semiclassical Thomas-Fermi (TF) approximation. In fact, this approximation averages out shell effects typical of finite nuclei and provides the overall energy dependence of the matrix elements. Within this context we follow Ref. [11], where semiclassical expressions for two-body matrix elements have been presented. In that reference a typical pairing matrix element, $\left\langle n \bar{n}|v| n^{\prime} \bar{n}^{\prime}\right\rangle$, where $|n\rangle$ is a single-particle shell-model state and $|\bar{n}\rangle$ is its time reversed counterpart, is averaged over a certain energy window, around the energy $E$,

$$
v(E)=\frac{1}{g^{2}(E)} \sum_{n}^{\prime} \sum_{n^{\prime}}^{\prime}\left\langle n \bar{n}|v| n^{\prime} \bar{n}^{\prime}\right\rangle,
$$

where the primes indicate that the summations run only over those states concentrated in a region $\pm \delta E$ around $E$. One has to normalize by the number of states in that energy interval, derived from the density of states $g(E)$ at energy $E$. As a window function we can for example adopt a Gaussian $\rho_{n}(E) \sim \exp \left[-\left(E-e_{n}\right)^{2} / \gamma^{2}\right]$, similarly to what is done in smoothing the level density according to the Strutinsky method (e.g., Ref. [2] Sec. 2.9.3). The width $\gamma$ should be taken of the order of the shell spacing to wash out the shell fluctuations. Conversely, it is well known that the Strutinsky averaging method is equivalent to the semiclassical TF method where one replaces the Gaussian by the normalized semiclassical density matrix at a given energy $E[11]$ :

$\rho_{E}=\frac{1}{g(E)} \delta\left(E-H_{c l}\right)=\frac{1}{g(E)} \delta\left[E-\frac{p 2}{2 m_{k}}-U(r)\right]$.

In the above relation the semiclassical level density $g$ is as follows:

$$
g(E)=\frac{1}{\pi} \int_{0}^{R_{c}} d r r^{2}\left(\frac{2 m_{k}}{\hbar^{2}}\right)^{3 / 2} \sqrt{E-U(r)}
$$

$R_{c}$ being the classical turning point. To make connection with the usual complete single-particle density matrix, we simply observe that it is obtained by integrating Eq. (3) over the energy up to the Fermi energy $e_{F}$ :

$$
\theta\left(e_{F}-H_{c l}\right)=\int_{0}^{e_{F}} d E g(E) \rho_{E} .
$$


In Eq. (4) we assume a constant value for the effective mass $m_{k}$, neglecting its possible dependence on position. The expression for the semiclassical diagonal matrix element of a two-body interaction $v\left(\vec{r}_{1}, \vec{r}_{2}\right)$ as a function of the single-particle energy is then given by the following [11]:

$$
\begin{aligned}
v(E)= & \int d^{3} r_{1} d^{3} r_{2} v\left(\vec{r}_{1}, \vec{r}_{2}\right) \int \frac{d^{3} p_{1} d^{3} p_{2}}{(2 \pi \hbar)^{6}} \frac{e^{\frac{i}{\hbar}\left(\vec{p}_{1}-\vec{p}_{2}\right) \vec{s}}}{g^{2}(E)} \\
& \times \delta\left[E-\frac{p_{1}^{2}}{2 m_{k}}-U\left(\frac{\vec{r}_{1}+\vec{r}_{2}}{2}\right)\right] \\
& \times \delta\left[E-\frac{p_{2}^{2}}{2 m_{k}}-U\left(\frac{\vec{r}_{1}+\vec{r}_{2}}{2}\right)\right]
\end{aligned}
$$

For an interaction that depends only on the magnitude of the relative coordinate $s \equiv\left|\vec{r}_{1}-\vec{r}_{2}\right|$, such as the Gogny interaction in the $S=0, T=1$ channel, one can integrate first over $d^{3} S$ and then over the momenta $d^{3} p_{1}$ and $d^{3} p_{2}$. We are then left with an integral over the center-of-mass $\vec{R}=\left(\vec{r}_{1}+\vec{r}_{2}\right) / 2$, obtaining the expression

$$
\begin{aligned}
v(E)= & c(E) \int d^{3} R[E-U(R)] \theta(E-U(R)) \\
& \times v\left(k_{E}(R), k_{E}(R)\right),
\end{aligned}
$$

where $v(k, k)$ is the on-shell pairing matrix element of the interaction $v(s)$ in the ${ }^{1} S_{0}$ channel and in a plane wave basis, evaluated at the local momentum $k=k_{E}(R)=$ $\frac{1}{\hbar} \sqrt{2 m_{k}[E-U(R)]}$, and $c(E)$ denotes the quantity

$$
c(E)=\frac{2 m_{k}^{3}}{4 \pi^{4} \hbar^{6} g^{2}(E)} .
$$

In the next section we calculate the pairing gap associated with the different interactions, and therefore we need the matrix elements between pairs of particles moving in time reversal states of different energies. For this purpose we employ a formula analogous to that given in Eq. (7), namely

$$
\begin{aligned}
v\left(E, E^{\prime}\right)= & \sqrt{c(E) c\left(E^{\prime}\right)} \int d^{3} R \sqrt{[E-U(R)]} \sqrt{\left[E^{\prime}-U(R)\right]} \\
& \times \theta[E-U(R)] \theta\left[E^{\prime}-U(R)\right] v\left(k_{E}(R), k_{E^{\prime}}(R)\right) .
\end{aligned}
$$

In this relation $E$ and $E^{\prime}$ indicate the energies of the singleparticle states of each pair.

We now return to the case of the induced interaction, associated with the matrix elements of Eq. (1). In this case the matrix elements depend separately on $\vec{r}_{1}$ and $\vec{r}_{2}$. We then integrate over the momenta first, obtaining for the diagonal matrix elements

$$
v_{\text {ind }}(E)=2 \sum_{n L M} c(E) \frac{\int d^{3} r_{1} d^{3} r_{2} f_{L n}\left(r_{1}\right) Y_{L M}\left(\hat{r}_{1}\right) f_{L n}\left(r_{2}\right) Y_{L M}^{*}\left(\hat{r}_{2}\right) j_{0}^{2}\left(k_{E}(R) s\right)[E-U(R)]}{E_{0}-2\left|E-e_{F}\right|-\hbar \omega_{n L}},
$$

where $j_{0}$ is the Bessel function. The index $n$ labels the different phonons of multipolarity $L$ (the phonons included in the present calculation are specified at the end of this section). Note that one can obtain numerical results from Eq. (10) by avoiding the six-dimensional integration. This is achieved by performing a multipole expansion of the quantity

$$
j_{0}^{2}(k s)[E-U(R)]=\sum_{l m} \frac{4 \pi}{2 l+1} F_{l}\left(r_{1}, r_{2} ; E\right) Y_{l m}\left(\hat{r}_{1}\right) Y_{l m}^{*}\left(\hat{r}_{2}\right),
$$

which leads to

$$
\begin{aligned}
v_{\text {ind }}(E)= & 2 \sum_{n L} 4 \pi c(E) \\
& \times \frac{\int d r_{1} d r_{2} r_{1}^{2} r_{2}^{2} f_{L n}\left(r_{1}\right) f_{L n}\left(r_{2}\right) F_{L}\left(r_{1}, r_{2} ; E\right)}{E_{0}-2\left|E-e_{F}\right|-\hbar \omega_{L n}} .
\end{aligned}
$$

In Ref. [11] the reliability of the $\mathrm{TF}$ approximation in reproducing the quantum mechanical $(\mathrm{QM})$ matrix elements has been checked in the particular case of the pairing matrix elements of a $\delta$ interaction acting among particles moving in a harmonic oscillator (HO) potential. Note that the TF approach implies an averaging over the quantum numbers associated with the different states belonging to the major shells and displaying an energy $E_{N}=\left(N+\frac{3}{2}\right) \hbar \omega$. The TF matrix elements have been compared with the averaged
QM matrix elements (taking degeneracies into account). Overall agreement was observed (cf. Table II of Ref. [11]).

A similar comparison for the pairing matrix elements of the Gogny force in the ${ }^{1} S_{0}$ channel is reported in Fig. 2. It is seen that the TF approach provides a good approximation to the average quantum matrix elements. However, the QM diagonal matrix elements, in particular those associated with $s$-states, are systematically larger (in absolute value) than the nondiagonal ones. One should be aware of the fact that the semiclassical density matrix, as already mentioned, can be considered to be the analog to the one obtained from a Strutinsky smoothing. Therefore, Eq. (3) implicitly represents a function of about $1 \hbar \omega$ width. Thus, the matrix element in Eq. (6) contains at the quantum level cross terms corresponding to at least one major shell. Taking, for example, the $2 s-1 d$ shell, we have to weigh the two diagonal matrix elements with the $2 s$ and $1 d$ wave-functions with a factor 1 and 25 , respectively. The nondiagonal $2 s-1 d$ element obtains the weight factor 10. Performing the arithmetic average yields the crosses of Fig. 2. In this way, we see that the TF expression [Eq. (6)] reproduces very well the quantal average. The more realistic case of the Gogny matrix elements in the Woods-Saxon potential (including spin-orbit) is illustrated in Fig. 3. The general pattern is similar to that shown in the previous figure. 


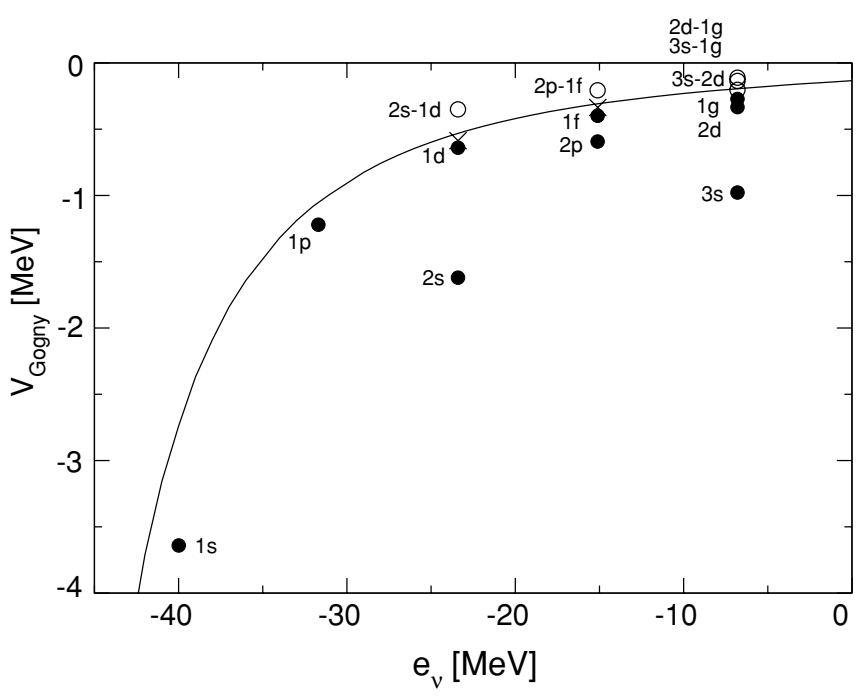

FIG. 2. Schematic model for the nucleus ${ }^{120} \mathrm{Sn}$. The semiclassical pairing matrix elements of the Gogny force (solid line) are compared to the quantal matrix elements for the case in which the single-particle wave functions have been calculated making use of a harmonic oscillator potential without the spin-orbit term (and $m_{k}=m$ ). The diagonal and nondiagonal matrix elements are denoted by filled and open circles respectively. The crosses correspond to the weighted averages of the matrix elements within a shell.

Having assessed its validity, we now employ the semiclassical approximation to compare the matrix elements of the induced interaction, the effective Gogny interaction, and a bare nucleon-nucleon interaction. Concerning the latter, the matrix elements of bare nucleon-nucleon interactions that contain a repulsive core display a qualitatively different momentum

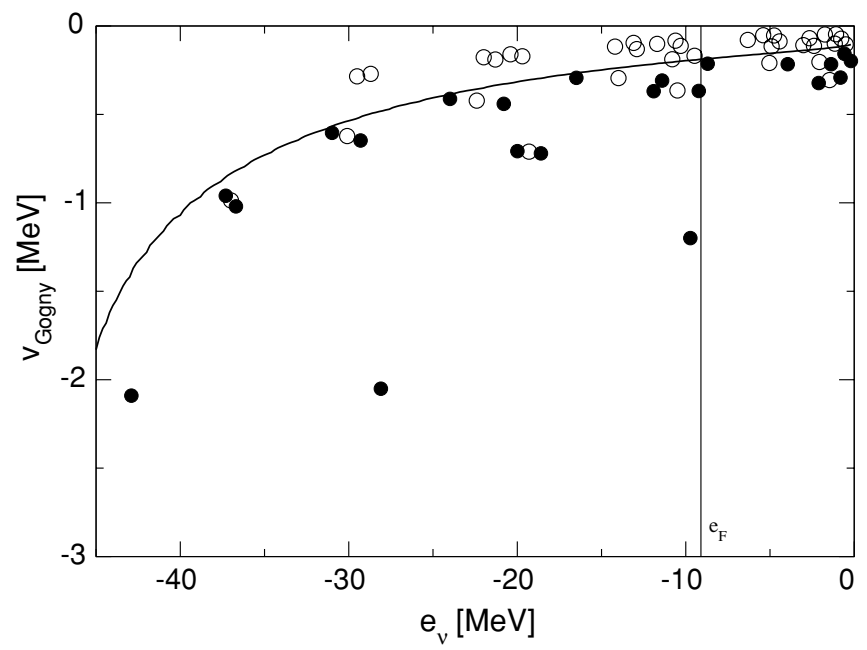

FIG. 3. The nucleus ${ }^{120} \mathrm{Sn}$. The same as Fig. 2 (i.e., also with $m_{k}=m$ ) but for the fact that the single-particle wave functions have been calculated making use of the Woods-Saxon potential including the spin-orbit term already used for Fig. 1, where the diagonal matrix elements have already been shown. The nondiagonal matrix elements are plotted here at an energy $e_{v}$, which is the average between the energies of the initial and final states.

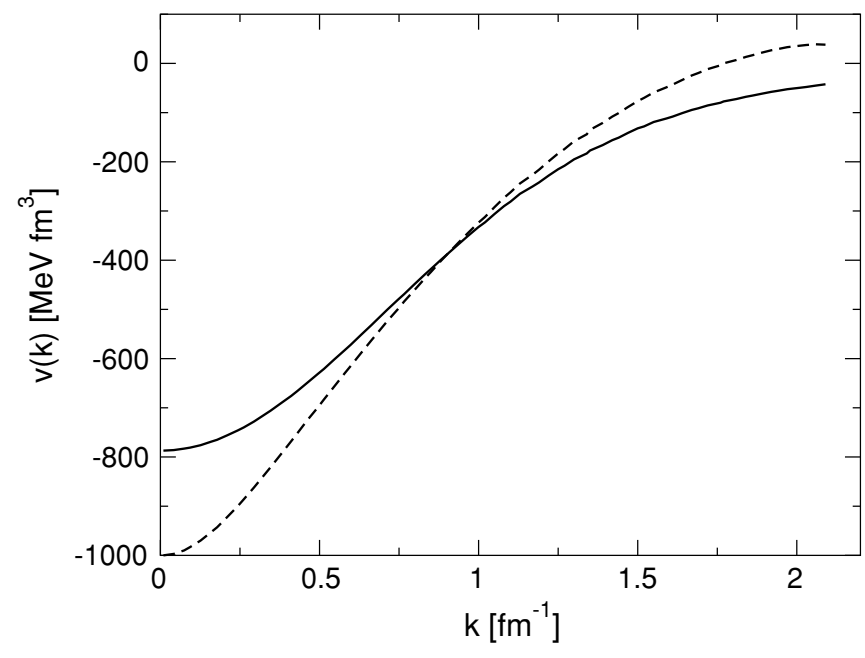

FIG. 4. The matrix elements of the Gogny (solid curve) and of the $v_{\text {low- } k}$ (dashed curve) interactions are plotted as a function of momentum.

dependence than that displayed by the Gogny interaction. We instead consider here the $v_{\text {low- } k}$ interaction, which has been devised to reproduce the low-momentum properties of the nucleon-nucleon force, including the experimental phase shifts, without the introduction of a repulsive core [12]. In particular, we employ a parametrization of $v_{\text {low- } k}$ devised to reproduce the properties of $v_{14}$ Argonne potential. This parametrization requires a momentum cutoff $k_{\text {cut }}$ equal to $2.1 \mathrm{fm}^{-1}$. The matrix elements of $v_{\text {low- } k}$ in a plane wave basis have been calculated in Ref. [13] and are reported in Fig. 4. It is seen that, whereas $v_{\text {Gogny }}$ is less attractive than $v_{\text {low- } k}$ for small momenta, it becomes more attractive for $k$ larger than $\approx 1 \mathrm{fm}^{-1}$.

The semiclassical matrix elements of the Gogny D1 interaction [Eq. (7)] and of the $v_{\text {low- } k}$ interactions calculated as a function of the single-particle energy for the nucleus ${ }^{120} \mathrm{Sn}$ are displayed in Fig. 5 (we remark that, close to the Fermi energy, the matrix elements of the Gogny interaction calculated with the D1S parametrization are less attractive by about $10 \mathrm{keV}$, as compared to the D1 parametrization used in the present paper). It is seen that the general trend is the same as that obtained in infinite matter, with the difference that the matrix elements of the two interactions look somewhat more similar to one another. Insight into this difference can be obtained with the help of Eq. (7). This relation is essentially an average of $v(k)$ calculated at the various local momenta in the nucleus. From Fig. 4 we see that in the interior of the nucleus (at larger local momenta) $v_{\text {Gogny }}$ is more attractive than $v_{\text {low- } k}$, but on the surface it tends to be less attractive than $v_{\text {low- } k}$.

The semiclassical matrix elements of the induced interaction are also shown in Fig. 5. These matrix elements are strongly peaked around the Fermi energy and reproduce rather well the average of the quantal results already shown in Fig. 1. In Fig. 6 we have separated the contributions arising from the exchange of phonons of multipolarities $L=2,3$, and 4 . We include all the phonons with energy below $30 \mathrm{MeV}$ in the calculation. The importance of the contributions with $L=2$ and 3 can be understood in terms of the high collectivity 


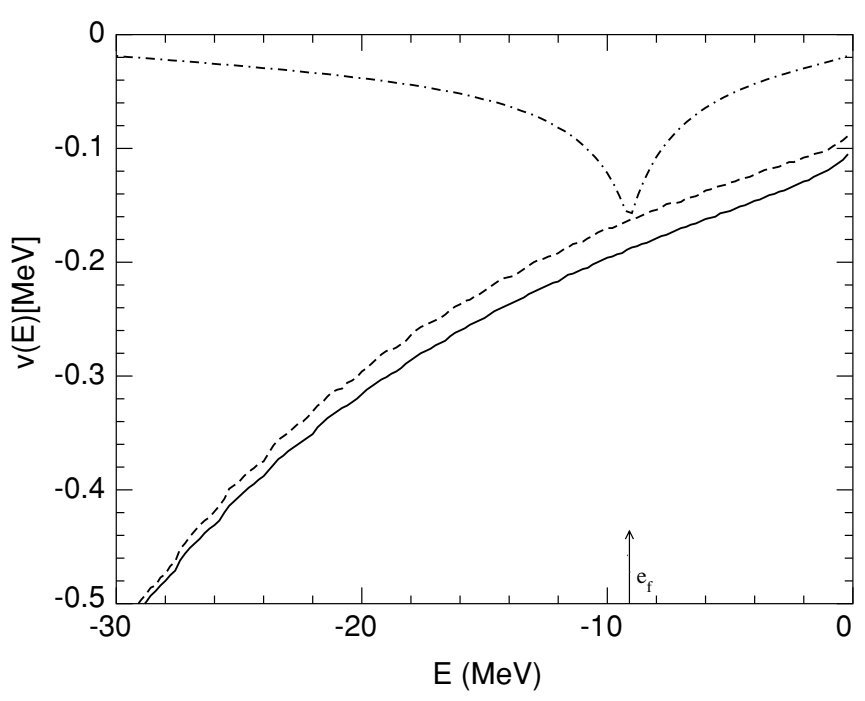

FIG. 5. The nucleus ${ }^{120} \mathrm{Sn}$. The semiclassical matrix elements of the induced interaction, calculated according to Eq. (11) (dash-dotted curve), are compared with the matrix elements of the Gogny force (solid curve, cf. Fig. 3) and with those of the $v_{\text {low- } k}$ interaction (dashed curve). Calculations are performed with $m_{k}=m$ and with the same Woods-Saxon potential used in Figs. 1 and 3.

and low energy of the associated collective modes. The energies and deformation parameters of the low-lying modes are $\hbar \omega_{2}=1.17 \mathrm{MeV}, \hbar \omega_{3}=2.42 \mathrm{MeV}, \hbar \omega_{4}=2.47 \mathrm{MeV}$ and $\beta_{2}=0.12, \beta_{3}=0.15, \beta_{4}=0.07$.

We also note that at the Fermi energy the matrix elements of $v_{\text {ind }}$ are rather large, of the order of those associated with the bare nucleon-nucleon potential $v_{\text {low- } k}$ in this connection, cf. the estimate in Ref. [14]).

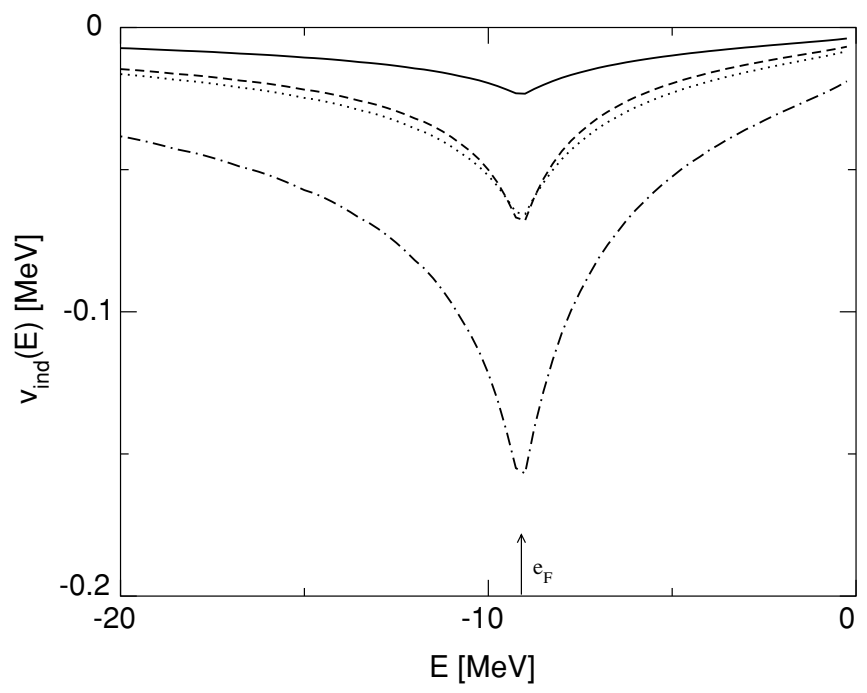

FIG. 6. The nucleus ${ }^{120} \mathrm{Sn}$. The semiclassical matrix elements of the induced interaction, as a function of the single-particle energy (calculated with $m_{k}=m$ ) associated with the multipolarities $L=2$, 3 , and 4 of the form factor [see Eq. (1)], are shown by a dashed, dotted, and solid line respectively. The sum of the three contributions is displayed by means of a dash-dotted line (cf. Fig. 5).

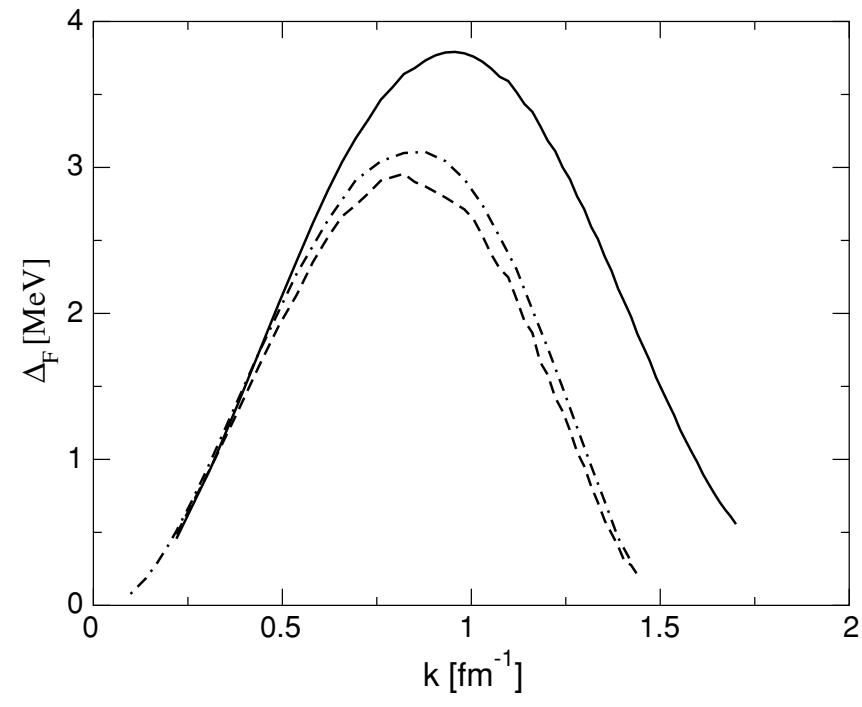

FIG. 7. Pairing gaps calculated in neutron matter as a function of the Fermi momentum, obtained with the Gogny interaction (solid line), the Argonne $v_{14}$ potential (dash-dotted line), and the $v_{\text {low- } k}$ potential (dashed line). The bare effective mass has been used in the calculation.

\section{PAIRING GAPS}

In this section, we discuss the results of calculations of the pairing gap that employ the matrix elements discussed above. In the nucleus under study, namely ${ }^{120} \mathrm{Sn}$, we obtain the state-dependent pairing gaps by solving the generalized BCS equations [15]: this means that we solve the HFB equations by treating the pairing sector self-consistently, whereas the mean field is described in terms of the WoodsSaxon potential already used in the calculation of the matrix elements. In this way, we take into account scattering processes between nucleons lying on orbits having different number of nodes/energies [5], associated with nondiagonal matrix elements of the type $v\left(E_{1}, E_{2} ; E_{1}^{\prime}, E_{2}^{\prime}\right)$, with different energies $E_{1}$ and $E_{2}$ (or $E_{1}^{\prime}$ and $E_{2}^{\prime}$ ). This is at variance with the usual BCS method in which the pairing gap receives contributions only from scattering processes between time-reversal states. We refer in the following either to quantal or to semiclassical calculations; in the latter case, this is done by solving the same equations as in the quantal case, but replacing the QM matrix elements with the TF ones, using Eq. (9) and the following prescription:

$$
E=\left(E_{1}+E_{2}\right) / 2, \quad E^{\prime}=\left(E_{1}^{\prime}+E_{2}^{\prime}\right) / 2 .
$$

The positive energy states are obtained by setting the system in a spherical box. We have checked that convergence is achieved using $R_{\mathrm{box}}=12 \mathrm{fm}$ and including all the states from the bottom of the potential up to the positive energy $E_{\text {cut }}=$ $50 \mathrm{MeV}$.

In infinite neutron matter, the pairing gaps calculated with the interactions $v_{\text {low- } k}$ and $v_{\text {Gogny }}$ are very similar up to Fermi momenta of the order of $0.6 \mathrm{fm}^{-1}$, becoming increasingly different from each other at higher momenta, as can be seen from Fig. 7 (cf. Ref. [6,13], where the D1S parametrization of the Gogny interaction has been used). The pairing gap obtained 


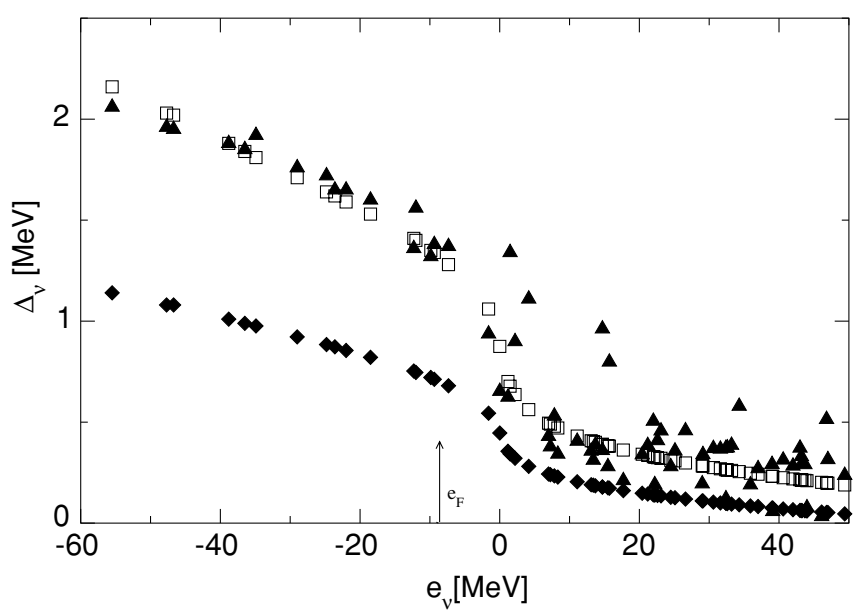

FIG. 8. State-dependent pairing gaps of ${ }^{120} \mathrm{Sn}$ calculated with a Woods-Saxon potential (with depth $V_{0}=-64 \mathrm{MeV}$, diffusivity $a=$ $0.65 \mathrm{fm}$, and radius $R_{0}=6.17 \mathrm{fm}$ ) as a function of the single-particle energy. The $k$-mass $m_{k}$ was set equal to $0.7 \mathrm{~m}$. The Fermi energy is $e_{F}=-8.6 \mathrm{MeV}$. Solid triangles (open squares) display the results of a HFB calculation with the Gogny interaction, with quantal (semiclassical) matrix elements. The solid diamonds refer instead to a HFB calculation using the semiclassical matrix elements of the $v_{\text {low- } k}$ potential.

with $v_{\text {low- } k}$ goes to zero, around saturation, as a function of $k_{F}$ much faster than that associated with $v_{\text {Gogny }}$; it can also be seen that the pairing gap obtained with $v_{\text {low- } k}$ reproduces quite accurately the result found allowing particles to interact through the Argonne $v_{14}$ bare $N N$ potential [16].

In the case of ${ }^{120} \mathrm{Sn}$, the pairing gap with the $v_{14}$ Argonne interaction and with $m_{k}=m$, was calculated previously in Ref. [17], obtaining a value of about $2.2 \mathrm{MeV}$ close to the Fermi energy. We have verified that essentially the same value is obtained with $v_{\text {low- } k}$, in keeping with the infinite matter case (cf. Fig. 7). In the following, however, we adopt the value $m_{k}=0.7 m$, which is the value associated with Hartree-Fock calculations performed with Gogny or Skyrme forces. In Fig. 8 we compare the state-dependent pairing gap obtained with the Gogny force and with the $v_{\text {low- } k}$ interaction. We also compare the pairing gaps obtained inserting the semiclassical pairing matrix elements in the HFB equations. We remark that the semiclassical approach takes out the scatter from shell effects, which is a desirable feature when one wishes to reveal generic trends.

The Gogny interaction leads to a pairing gap of $1.4 \mathrm{MeV}$ close to the Fermi energy, in good agreement with the experimental value deduced from the odd-even mass difference. This value is about two times larger than that obtained with $v_{\text {low- } k}$. Note also that the pairing gap obtained with $v_{\text {low- } k}$ reproduces the results obtained with the bare $v_{14}$ interaction [8].

\footnotetext{
${ }^{1}$ We remark that using the D1S parametrization of the Gogny force, instead of D1, one obtains a gap at the Fermi energy of about $1.2 \mathrm{MeV}$.
}

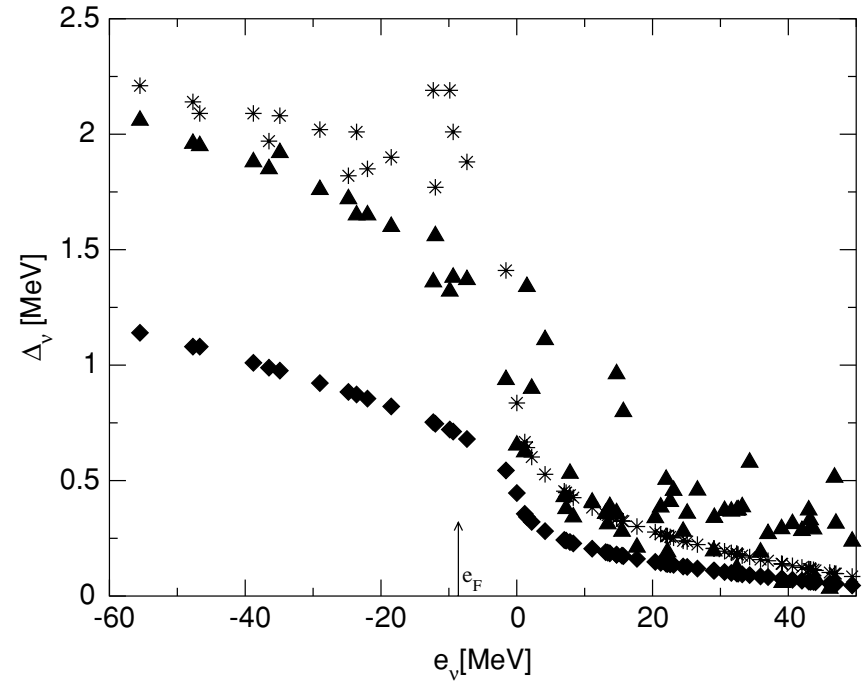

FIG. 9. State-dependent pairing gap of ${ }^{120} \mathrm{Sn}$ obtained making use of the Woods-Saxon potential used in calculating the results displayed in Fig. 8 (i.e., with $m_{k}=0.7 \mathrm{~m}$ ) and different interactions. Diamonds and triangles show again the gaps obtained with the $v_{\text {low- } k}$ and $v_{\text {Gogny }}$ interactions, respectively. Stars show the gap associated with the matrix elements obtained, summing the matrix elements of $v_{\text {low- } k}$ and $v_{\text {ind. }}$. The latter have been evaluated using Eq. (1).

In Fig. 9 we show the state-dependent pairing gaps obtained with the pairing matrix elements of $v_{\text {total }}=v_{\text {low- } k}+v_{\text {ind }}$, resulting from the sum of the matrix elements of the bare interaction $v_{\text {low- } k}$ and of the induced interaction $v_{\text {ind }}$. The average value of the resulting pairing gap $\Delta_{\text {total }}$ at the Fermi energy is about $2 \mathrm{MeV}$. This value is about $30 \%$ higher than that obtained in Ref. [8], where we solved the Dyson-Gor'kov equation, taking properly into account all polarization effects (induced interaction, self-energy, and vertex corrections) [18].

This is consistent with the fact that, according to nuclear field theory (NFT) [19], if one considers the effects of the exchange of phonons between pairs of nucleons, one has to consider at the same time processes where the phonon is absorbed by the same nucleon that has virtually excited it. Such processes lead to self-energy ( $\omega$-mass and single-particle splitting [10]) as well as to vertex correction phenomena (see Appendix C). Of these processes and for the nucleus under discussion $\left({ }^{120} \mathrm{Sn}\right), \omega$-mass effects are the most important [8]. Taking such effects into account is equivalent to use a residual interaction $v_{\text {ren }}=v_{\text {total }} Z_{\omega}$, where $Z_{\omega}=\left(m_{\omega} / m\right)^{-1}$ is the quasiparticle strength [10] at the Fermi energy (cf. Appendix A). Consequently $\Delta_{\text {ren }}=Z_{\omega} \Delta_{\text {tot }}$ Refs. [20,22,23]. Because $v_{\text {ind }}$ is proportional to $\beta_{L}^{2} / D E N_{L}$, that is, to the square of the deformation parameters associated with the low-lying collective vibration and inversely proportional to the energy denominator $D E N_{L}$ appearing in Eq. (1), a typical error of $20 \%$ on $\beta_{L}$ and $D E N_{L}$ implies a $60 \%$ error in $v_{\text {ind }}$. Making use of this fact and of the results of Figs. 5 and 9 one obtains, as discussed in Appendix B, $\Delta_{\text {ren }} \approx Z_{\omega} \Delta_{\text {tot }} \approx 1.3 \pm 0.14 \mathrm{MeV}$. It is seen that the $60 \%$ error in $v_{\text {ind }}$ has been reduced to a $10 \%$ in $\Delta_{\text {ind }}$. This is because 
the stronger the particle-vibration coupling is, the larger the contribution to $\Delta_{\text {tot }}$ associated with the induced interaction but the smaller the amount of single-particle content of levels around $e_{F}$ (and thus the smaller $Z_{\omega}$ ), and vice versa. The self-consistency between collectivity of the modes, strength of the particle-vibration coupling, and quasiparticle residue at the pole, typical of NFT, allows theory to make predictions that are more accurate than the basic parameters entering the calculation, as a result of a delicate process of cancelation of errors [20].

\section{CONCLUSIONS}

Summing up, we have found that the small difference existing between the matrix elements of the bare $v_{\text {low- } k}$ interaction and those of the Gogny interaction leads to important differences between the pairing gaps associated with the two forces $\left(\Delta_{\text {Gogny }} \approx 2 \Delta_{\text {low- } k}\right.$, for $\left.m_{k} \approx 0.7 \mathrm{~m}\right)$. This difference is removed and eventually overwhelmed by including the attractive contribution coming from the induced interaction $v_{\text {ind }}$ arising from the exchange of surface vibrations, which acts only on a rather small energy range around $e_{F}$. We note that the pairing gap in ${ }^{120} \mathrm{Sn}$ is not much changed, including also the effect of spin fluctuations [23], which instead give the dominant (repulsive) contribution to the induced interaction in neutron matter.

The $25 \%$ excess displayed by the resulting total pairing gap with respect to the experimental value is corrected by considering the corresponding quasiparticle strength at the Fermi energy. An important question that remains to be addressed quantitatively is the ability $v_{\text {low- } k}+v_{\text {ind }}$ as well as $v_{\text {Gogny }}$ have to describe in detail the isotopic and nuclear structure dependences displayed by the pairing gap $\Delta_{\exp }$ derived from the experimental odd-even mass differences. On one hand, both the Gogny force and the induced interaction display a typical overall dependence on the nuclear mass number [24,25]. On the other hand, on a finer scale, the induced interaction depends on the specific properties of the low-lying collective vibrational states of the individual nuclei and thus on the details of the structure of each single nucleus.

Within this context, we note that the dependence of the pairing gaps generated by $v_{\text {Gogny }}$ and by $v_{\text {low- } k}+v_{\text {ind }}$ on temperature and rotational frequency is expected to be quite different, as $v_{\text {Gogny }}$ and $v_{\text {low- } k}$ are essentially independent of these parameters, whereas $v_{\text {ind }}$ is quite sensitive to them. ${ }^{2}$

\section{ACKNOWLEDGMENTS}

We thank L. Coraggio for providing us with the matrix elements of the $v_{\text {low- } k}$ interaction.

\footnotetext{
${ }^{2}$ Within this context we note that in a recent study in symmetric nuclear matter a strong attraction arising from the induced interaction has been found [26]. How this result relates to the present discussion on finite nuclei is still an open question.
}

\section{APPENDIX A}

\section{A. Simple estimate of the pairing gap}

In what follows we consider some of the consequences the particle-vibration coupling has on the pairing correlations of particles moving in a single $j$-shell interacting through a bare nucleon-nucleon pairing potential with constant matrix elements $G$ [20].

For this simple model, the value of the occupation numbers $U_{v}$ and $V_{v}$ must be the same for all the $2 j+1$ orbitals. In particular, the occupation probability for the case when the system is occupied with $N=\Omega$ particles (half-filled shell), where

$$
\Omega=\frac{2 j+1}{2}
$$

is

$$
\begin{gathered}
V=\sqrt{\frac{N}{2 \Omega}}=\sqrt{\frac{1}{2}}, \\
U=\sqrt{1-\frac{N}{2 \Omega}}=\sqrt{\frac{1}{2}} .
\end{gathered}
$$

Consequently, the pairing gap is given by the following relation:

$$
\Delta=G \sum_{v>0} U_{v} V_{v}=\frac{G \Omega}{2} .
$$

Because the density of levels is proportional to the $\omega$-mass $\left(m_{\omega}\right)$, the effective (dressed) degeneracy can be written as follows:

$$
\Omega_{\mathrm{eff}}=\frac{\Omega}{Z_{\omega}}
$$

in terms of the (bare) single-particle degeneracy $\Omega$ and of the quasiparticle strength at the Fermi energy $Z_{\omega}=\left(m_{\omega} / m\right)^{-1}$, where $m_{\omega}=m(1+\lambda)$ and $\lambda$ is the mass enhancement factor [27]. In the case of nuclei this dimensionless quantity (which measures the strength with which nucleons couple to low-lying collective vibrations) is of the order of 0.5 , closer to the strong than to the weak coupling situation of BCS (in which case $\Delta \sim \lambda$ ) [22], cf. Eq. (C19).

Because of their coupling to vibrations, nucleons spend part of the time in more complicated configurations than pure single-particle states. The quantity (quasiparticle strength [10]) $Z_{\omega}=(1+\lambda)^{-1} \approx 0.7$ measures the content of single-particle strength present in levels around the Fermi energy available for nucleons to interact through a two-body interaction, in particular through a pairing force. Consequently, because of the self-energy processes arising from the particle-vibration coupling phenomenon, the pairing strength becomes $G Z_{\omega}{ }^{2}$.

The exchange of vibrations between pairs of nucleons moving in time reversal states close to the Fermi energy gives rise to an effective pairing interaction of strength $g_{p-v} Z_{\omega}^{2}$, where $g_{p-v}$ stands for the induced pairing interaction controlled by the particle-vibration coupling vertices. Taking these effects into account, one can write

$$
\Delta_{\text {ren }}=\frac{Z_{\omega}}{2}\left(G+g_{p-v}\right) \Omega \text {. }
$$




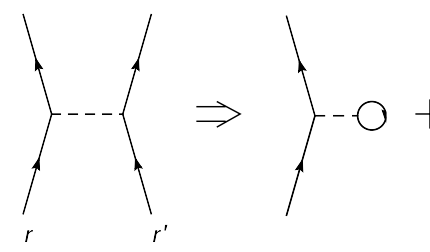

(a)

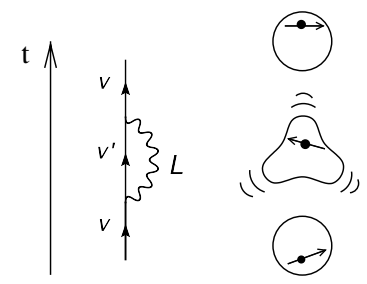

(d)

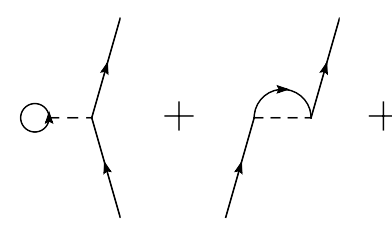

(b)
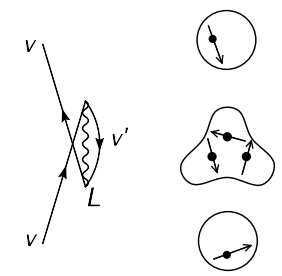

(e)
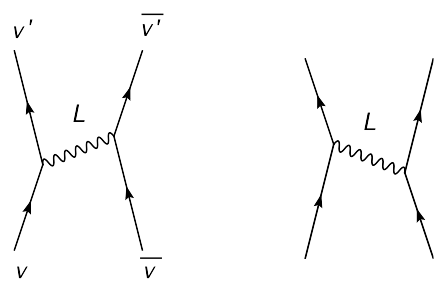

(f)

FIG. 10. Nucleons interact in a scattering experiment through the bare $N N$-interaction (a), the analysis of the associated differential cross section being made in terms of phase shifts. The main effect of this bare two-body interaction acting among nucleons inside the nucleus is to produce a mean field, sum of a local (b) (Hartree) and a non-local (c) (Fock) potential. In this potential, nucleons move independently of each other feeling the pullings and pushings of the other nucleons only when they try to leave the nucleus. In other words, they move in a singleparticle potential produced by all the other nucleons bouncing elastically off the surface. However, this surface can vibrate as a whole, as testified by the existence of low-lying collective surface modes and of giant resonances in the inelastic spectrum of nucleus-nucleus collisions. Thus, from time to time a nucleon can bounce inelastically off the nuclear surface, setting it into vibration, changing state of motion and, at a later time, reabsorb the vibration returning to the original state as shown in (d). A similar process, but this time associated with the virtual excitation of the vacuum $\left(\mathrm{e}^{\prime}\right)$ and with the Pauli principle is at the basis of the process depicted in (e), obtained from a simple change in the time ordering from process (d). A vibration excited by a nucleon can be reabsorbed by a second nucleon (f). Such a process leads to an induced interaction among nucleons, associated with the polarization of the nuclear medium.

Identifying $G$ with $v_{\text {low }-k}, g_{p-v}$ with $v_{\text {ind }}$, and $\Omega$ with the (bare, $m_{k} \approx 0.7 \mathrm{~m}$ ) degeneracy, one finds from Fig. 9 ,

$$
\frac{1}{2}\left(G+g_{p-v}\right) \Omega \approx 2 \mathrm{MeV} .
$$

As seen from Eq. (A6), the number to be compared with experimental findings is

$$
\Delta_{\text {ren }}=Z_{\omega} \times 2 \mathrm{MeV} \approx 1.4 \mathrm{MeV}
$$

Note that in the above discussion we have not considered the errors to be expected in estimating both $g_{p-v}$ and $Z_{\omega}$. This subject is taken up in Appendix B.

\section{APPENDIX B}

\section{B. Simple estimate of $\lambda$ and $Z_{\omega}$}

From Fig. 5 it is seen that the average values of $v_{\text {ind }}$ and $v_{\text {low }-k}$ at the Fermi energy are $\left\langle v_{\text {ind }}\right\rangle=-0.15 \mathrm{MeV}$ and $\left\langle v_{\text {low- } k}\right\rangle=-0.16 \mathrm{MeV}$. Because $v_{\text {ind }}$ is of the order of $\sum_{L} \beta_{L}^{2} / D E N_{L}$, the matrix elements of $v_{\text {ind }}$ will display an error equal to twice the average error displayed by $\beta_{L}$ plus that displayed by $D E N_{L}$. Assuming this error to be $20 \%$ for both quantities (a rather extreme negative situation) one concludes that $\left\langle v_{\text {ind }}\right\rangle=0.15 \pm 0.09 \mathrm{MeV}$. Consequently $\left\langle v_{\text {tot }}\right\rangle=$ $\left\langle v_{\text {ind }}\right\rangle+\left\langle v_{\text {low- } k}\right\rangle=-0.31 \pm 0.09 \mathrm{MeV}$. Because $\Delta_{\text {total }}=$ $2 \mathrm{MeV}$ for $\left\langle v_{\text {tot }}\right\rangle=-0.31 \mathrm{MeV}$, one obtains $\Delta_{\text {total }}=(2 \pm$ 0.6) $\mathrm{MeV}$.

We now proceed to the calculation of $\lambda=N(0)\left\langle v_{\text {ind }}\right\rangle$, where $N(0)$ is the density of single-particle levels around the Fermi energy for one spin orientation and one type of nucleon. Making use of the value $N(0)=3.4 \mathrm{MeV}^{-1}$ appropriate for ${ }^{120} \mathrm{Sn}$, one gets $\lambda=0.5 \pm 0.3$. Consequently, $\Delta_{\text {ren }}=Z_{\omega} \Delta_{\text {tot }}=1.30 \pm 0.14 \mathrm{MeV}$, where $Z_{\omega}=(1+\lambda)^{-1}$. We thus note a conspicuously smaller error for $\Delta_{\text {ren }}$ than for $v_{\text {ind }}$. This is because the larger is $\lambda$ (induced pairing contribution), the smaller is the content of single-particle strength (and thus $Z_{\omega}$ ) associated with levels lying close to the Fermi energy and vice versa.

\section{APPENDIX C}

As seen from the graphical treatment of the processes induced by the $N N$ interaction acting among nucleons moving in the atomic nucleus [cf. Fig. 10(a)-(e)], the Hamiltonian describing the single-particle motion reads (Ch. 9 of Ref. [20])

$$
H_{\mathrm{sp}}=\left[-\frac{\hbar^{2}}{2 m} \nabla^{2}+U_{x}(k)+\Delta E(\omega)\right]+U_{H}(r)+i W(r),
$$

where $U_{H}(r)$ [cf. Fig. 10(b)] and $U_{x}(k)$ [cf. Fig. 10(c)] are the Hartree- and the Fock-potential. The dependence of $U_{x}$ on the momentum of the particle is associated with the nonlocality arising from the Pauli principle and from the momentum dependence of the force. The dependence of the real part of the 
single-particle self-energy $\Delta E$ on the frequency is associated with the nonlocality in time generated by the coupling to surface vibrations excited by the particle at a given time and reabsorbed at a different time [virtual, off-the-energy shell process, cf. Figs. 10(d) and 10(e)]. Effects associated with real, energy-shell processes are described by the imaginary part of the single-particle self-energy $W(r)$.

For many purposes, it is possible to rewrite the term in square brackets in Eq. (C1) as a kinetic energy term with an effective mass $m^{*}$ (e.g., Refs. [10,20]):

$$
H_{\mathrm{sp}}=\frac{\hbar^{2}}{2 m^{*}} \nabla^{2}+\tilde{U}+i \tilde{W},
$$

which is the optical model with $\tilde{U}=\left(\mathrm{m} / \mathrm{m}^{*}\right) U_{H}$ and $\tilde{W}=$ $\left(\mathrm{m} / \mathrm{m}^{*}\right) W$. Note that $W=0$ for single-particle levels around the Fermi energy $\left(\left|e_{v}-e_{F}\right| \leqslant 5 \mathrm{MeV}\right)$ and acquires a finite value only when real transitions are possible (i.e., for $\mid e_{v}-$ $e_{F} \mid \gg 5 \mathrm{MeV}$ ).

The effective mass

$$
\frac{m^{*}}{m}=\frac{m_{\omega}}{m} \frac{m_{k}}{m}
$$

is the product of the $k$ mass

$$
m_{k}=m\left(1+\frac{m}{\hbar^{2} k} \frac{\partial U_{x}}{\partial k}\right)^{-1}
$$

and of the $\omega$ mass

$$
m_{\omega}=m\left(1-\frac{\partial \Delta E}{\partial \omega}\right) .
$$

Note that $m^{*}$ depends on $r$, as a result of the combined $r$ dependence of $m_{k}$ (inversely proportional to a function of the density in the case of the Skyrme interaction) and $m_{\omega}$ (peaked at the nuclear surface) [10]. In what follows we use typical average values of both $m_{k}$ and $m_{\omega}$.

The contribution to the real part of the self-energy by the process shown in Fig. 10(d) (polarization term) can be written

$$
\Delta E(\omega)=\sum_{\nu>0} \frac{V^{2}\left(\nu, v^{\prime} ; L\right)}{\omega-\left(e_{\nu^{\prime}}+\hbar \omega_{L}\right)},
$$

where $V\left(v, v^{\prime} ; L\right)$ is the matrix element of the particlevibration coupling Hamiltonian, $\omega$ is the energy of the singleparticle state measured with respect to $e_{F}$, and $e_{v^{\prime}}=e_{v^{\prime}}-e_{F}$. One can thus write

$$
m_{\omega}=m\left(1-\frac{\partial \Delta E}{\partial \omega}\right)_{\omega=0}=m(1+\lambda),
$$

where

$$
\left(\frac{\partial \Delta E}{\partial \omega}\right)_{\omega=0}=-\sum_{\nu>0} \frac{V^{2}\left(\nu, v^{\prime} ; L\right)}{\left(e_{\nu^{\prime}}+\hbar \omega_{L}\right)^{2}} .
$$

Approximating the sum over the single-particle levels by an integral and calling $N(0)$ the density of single-particle levels at the Fermi energy (for one spin orientation), one can write

$$
\left(\frac{\partial \Delta E}{\partial \omega}\right)_{\omega=0} \approx-N(0) \int_{0}^{\infty} \frac{V^{2} d e}{\left(e+\hbar \omega_{L}\right)^{2}}=-N(0) \frac{V^{2}}{\hbar \omega_{L}},
$$

where for simplicity a constant particle-vibration matrix element $V$ has been assumed. The core correlation part of the self-energy (see Fig. 10(e)) gives an equal contribution. Consequently, the total value of $(\partial \Delta E / \partial \omega)_{\omega=0}$, sum of polarization and core contributions, is given by Eq. (C9) but multiplied by a factor of 2. Making use of this result and of Eq. (C7) one can write the mass enhancement factor [27] as

$$
\lambda=N(0) g_{p-v},
$$

where

$$
g_{p-v}=\frac{2 V^{2}}{\hbar \omega_{L}}
$$

is the particle-vibration coupling parameter.

Because $N(0) \sim m^{*}=m_{k} m_{\omega} / m$, where $m_{k} \approx 0.7 m$ (essentially independent of the single-particle energy) and $m_{\omega}=$ $1.5 m$ over an energy range of about $\pm 5 \mathrm{MeV}$ around the Fermi energy (that is, of the order of the energy of the low-lying collective surface vibrations), becoming equal to the bare mass for single-particle levels with $\left|e_{v}-e_{F}\right| \gg 5 \mathrm{MeV}$ [10], one can understand why the empirical evidence concerning the energy of single-particle levels around the Fermi energy is well described by the motion of nucleons in a real, energyindependent potential, with a mass equal to the bare nucleon mass. However, the situation is in fact more subtle than that described by such a simple model. In particular, because of its coupling to the nuclear surface, a particle that starts in a pure single-particle configuration is forced to more complicated configurations [cf. Figs. 10(d) and 10(e)]. Consequently, the probability of finding a particle in a single-particle state below the Fermi level is smaller than one. Similarly, an empty state lying above the Fermi energy becomes partially occupied. The quasiparticle strength [10]

$$
Z_{\omega}=\left(\frac{m_{\omega}}{m}\right)^{-1},
$$

gives a measure of the occupation factor associated with levels close to the Fermi energy $(\approx 0.7)$. Another basic difference between the Hamiltonian (C2) (with $\tilde{W}=0$ ) and the standard Hamiltonian $H_{0}=\hbar^{2} / 2 m+U_{H}$, is associated with the dependence of $N(0)$ on the rotational frequency and on temperature. This is because for $\hbar \omega_{\text {rot }}$ and/or $T$ of the order of $\hbar \omega_{L}$ (i.e., about $1-2 \mathrm{MeV}$ ), low-lying collective vibrations are strongly modified and $\lambda$ essentially vanishes. This means that, for strongly rotating and/or highly excited nuclei, $m^{*} \approx m_{k}$, a fact which leads to a strong decrease of the density of levels, as compared to that observed when the nucleus is close to its ground state. This has important consequences, among other things, for the cooling of highly excited, strongly rotating nuclei, in particular concerning the decay of the giant dipole resonance (cf. [28] and refs. therein).

The vibration excited by a nucleon interacting with the surface can be absorbed by a second nucleon, as shown in Fig. 10(f), giving rise to an induced interaction. In particular to an interaction that contributes to pairing. Nucleons in time-reversed states with energies $e$ exchange a phonon of 
multipolarity $L$ and make a transition to final states $\left|v^{\prime}\right\rangle$ and $\left|\bar{v}^{\prime}\right\rangle$ with energies $e^{\prime}$. The symmetrized matrix element of the pairing-induced interaction [with respect to initial and final states, and not with respect to the energy of the two interacting single-particle states as done in Eq. (1), where the Bloch-Horowitz approach is used, whereas here, for simplicity, the Rayleigh-Schrodinger prescription is employed] can be written as

$$
\begin{aligned}
v_{\nu v^{\prime}} & =\frac{1}{2}\left[2 \frac{V^{2}\left(\nu, v^{\prime} ; L\right)}{e_{v}-\left(e_{\nu^{\prime}}+\hbar \omega_{L}\right)}+2 \frac{V^{2}\left(\nu, v^{\prime} ; L\right)}{e_{\nu^{\prime}}-\left(e_{v}+\hbar \omega_{L}\right)}\right] \\
& =\frac{2 \hbar \omega_{L} V^{2}\left(\nu, v^{\prime}, L\right)}{\left(e_{v}-e_{\nu^{\prime}}\right)^{2}-\left(\hbar \omega_{L}\right)^{2}},
\end{aligned}
$$

where the factor of two accounts for the contribution of the two time orderings whereas the factor $1 / 2$ averages between initial and final states contribution. For $e_{v} \approx e_{v^{\prime}} \approx e_{F}$ and assuming again a constant particle-vibration coupling matrix element $V$, it can be approximated with

$$
v_{v v^{\prime}}=-\frac{2 V^{2}}{\hbar \omega_{L}}=-g_{p-v} .
$$

Making use of typical values of $\lambda \approx 0.5$, and of $N(0) \approx$ 3.4 $\mathrm{MeV}^{-1}$, appropriate for ${ }^{120} \mathrm{Sn}$, one obtains [cf. Eq. (C10)] $\left|v_{v v^{\prime}}\right| \approx 0.15 \mathrm{MeV}$, a value that is of the same order of magnitude as that of the empirical pairing matrix element $G=$ 25/A MeV [14,21].

From the above discussion one can conclude that although the bare nucleon-nucleon interaction is essential for the production of pair correlations in nuclei, the induced interaction because of phonon exchange also contributes in a consistent fashion to these correlations. To assess the importance of the induced interaction we estimate the associated pairing gap (neglecting the bare interaction completely), making use of the $\mathrm{BCS}$ equation

$$
\Delta=g_{p-v} \sum_{v>0} \frac{\Delta}{2 E_{v}}
$$

Approximating the sum by an integral this relation can be written as

$$
\begin{aligned}
1 & =g_{p-v} N(0) \int_{-\omega_{D}}^{\omega_{D}} d e \frac{1}{2 \sqrt{e^{2}+\Delta^{2}}} \\
& =g_{p-v} N(0) / \operatorname{arcsinh}\left(\frac{\omega_{D}}{\Delta}\right),
\end{aligned}
$$

where $\omega_{D}$ is a typical energy associated with low-lying collective nuclear vibrations. From the relation given above one obtains

$$
\Delta=\omega_{D} / \sinh \left(\frac{1}{\lambda}\right)
$$

where use was made of the definition (C10). In the case in which $\lambda \ll 1$ (weak coupling limit) one can write

$$
\Delta=2 \omega_{D} \exp \left(-\frac{1}{\lambda}\right)
$$

whereas

$$
\Delta=\omega_{D} \lambda
$$

in the case in which $\lambda \gg 1$ (strong coupling limit). Arguably, a better characterization of the weak and strong coupling regimes is provided by the relations $\lambda^{2} \ll \lambda$ and $\lambda^{2} \geqslant \lambda$. Because in the nuclear case $\lambda \approx 0.4-0.6$, one finds within this context an intermediate situation. Making use of this range of values of $\lambda$ and of a typical value of $\omega_{D} \approx 1.5-2 \mathrm{MeV}$, one obtains from the above equations values of $\Delta$ that are of the same order of magnitude of the empirical value $12 / \sqrt{A} \mathrm{MeV}$ [14]. This result strongly indicates the need to consider on equal footing the induced pairing interaction and the bare $N N$ interaction, in a quantitative description of pairing correlations in nuclei. From a more basic point of view, and as explicitly shown by the close relation existing among $m^{*}, Z_{\omega}, N(0)$, and $\Delta$ (all simple functions of $\lambda$ ), the processes displayed in Fig. 10 have to be considered simultaneously in a consistent field theoretical description of the single-particle and collective degrees of freedom of atomic nuclei and of their interweaving [19]. A recent example of such a treatment can be found in Ref. [8].
[1] J. P. Blaizot and G. Ripka, Quantum Theory of Finite Systems (The MIT Press, Cambridge, Mass., 1985).

[2] P. Ring and P. Schuck, The Nuclear Many-Body Problem (Springer-Verlag, New York, 1980).

[3] J. Dechargé and D. Gogny, Phys. Rev. C 21, 1568 (1980).

[4] Y. Yu and A. Bulgac, Phys. Rev. C 65, 51305(R) (2002); Phys. Rev. Lett. 90, 222501 (2003).

[5] T. Duguet, P. Bonche, P.-H. Heenen, and J. Meyer, Phys. Rev. C 65, 014311 (2002); J. Dobaczewski, W. Nazarewicz, T. R. Werner, J. F. Berger, C. R. Chinn, and J. Dechargé, ibid. 53, 2809 (1996).

[6] E. Garrido, P. Sarriguren, E. Moya de Guerra, and P. Schuck, Phys. Rev. C 60, 064312 (1999); E. Garrido, P. Sarriguren, E. Moya de Guerra, U. Lombardo, P. Schuck, and H. J. Schulze, ibid. 63, 037304 (2001).
[7] U. Lombardo and H.-J. Schultze, in Physics of Neutron Star Interiors, edited by D. Blaschke, N. K. Glendenning, and A. Sedrakian (Springer-Verlag, Berlin, 2001), p. 30.

[8] F. Barranco, R. A. Broglia, G. Colò, E. Vigezzi, and P. F. Bortignon, Eur. Phys. J. A 21, 57 (2004).

[9] F. Barranco, R. A. Broglia, G. Gori, E. Vigezzi, P. F. Bortignon, and J. Terasaki, Phys. Rev. Lett. 83, 2147 (1999).

[10] C. Mahaux, P. F. Bortignon, R. A. Broglia, and C. H. Dasso, Phys. Rep. 120, 1 (1985).

[11] X. Viñas, P. Schuck, M. Farine, and M. Centelles, Phys. Rev. C 67, 054307 (2003).

[12] L. Coraggio, A. Covello, A. Gargano, N. Itaco, and T. T. S. Kuo, Phys. Rev. C 66, 064311 (2002).

[13] A. Sedrakian, T. T. S. Kuo, H. Müther, and P. Schuck, Phys. Lett. B576, 68 (2004). 
[14] A. Bohr and B. R. Mottelson, Nuclear Structure (Benjamin, New York, 1975), Vol. II, p. 432.

[15] F. Barranco, R. A. Broglia, H. Esbensen, and E. Vigezzi, Phys. Rev. C 58, 1257 (1998).

[16] A. Schwenk, B. Friman, and G. E. Brown, Nucl. Phys. A713, 191 (2003).

[17] F. Barranco, R. A. Broglia, H. Esbensen, and E. Vigezzi, Phys. Lett. B390, 13 (1997).

[18] J. Terasaki, F. Barranco, R. A. Broglia, E. Vigezzi, and P. F. Bortignon, Nucl. Phys. A697, 127 (2002).

[19] D. R. Bes, R. A. Broglia, G. G. Dussel, R. J. Liotta, and H. M. Sofia, Nucl. Phys. A260, 1 (1976); A260, 27 (1976); D. R. Bes, R. A. Broglia, G. G. Dussel, R. J. Liotta, and R. J. Perazzo, ibid. A260, 77 (1976).

[20] D. Brink and R. A. Broglia, Nuclear superfluidity: pairing in finite systems (Cambridge University Press, Cambridge, 2005).
[21] A. Bohr and B. R. Mottelson, Nuclear Structure (Benjamin, New York, 1969), Vol. I.

[22] J. R. Schrieffer, Superconductivity (Benjamin, New York, 1964).

[23] G. Gori, F. Ramponi, F. Barranco, P. F. Bortignon, R. A. Broglia, G. Colò, and E. Vigezzi, Phys. Rev C 72, 011302(R) (2005).

[24] S. Hilaire, J.-F. Berger, M. Girod, W. Satula, and P. Schuck, Phys. Lett. B531, 61 (2002).

[25] N. Giovanardi, F. Barranco, R. A. Broglia, and E. Vigezzi, Phys. Rev. C 65, 041304(R) (2002).

[26] C. Shen, U. Lombardo, and P. Schuck, Phys. Rev. C 71, 054301 (2005).

[27] G. Grimvall, The Electron-Phonon Interaction in Metals (NorthHolland, Amsterdam, 1981).

[28] P. F. Bortignon, A. Bracco, and R. A. Broglia, Giant Resonances: Nuclear Structure at Finite Temperature (Harwood Academic Press, New York, 1998). 